\title{
A REMARK ON HORROCKS' THEOREM ABOUT PROJECTIVE $A[T]$-MODULES
}

\author{
BUDH S. NASHIER
}

\begin{abstract}
A projective module $P$ over $A[T]$ needs no more generators than $P_{f}$ for a monic $f$, and it is nearly determined by $P_{f}$.
\end{abstract}

The rings we consider here are assumed to be commutative with identity and the modules finitely generated. For a module $M$ over a ring $R$, we let $\mu(M)$ denote the least number of elements in $M$ required to generate $M$ as an $R$-module.

Horrocks' Theorem [1] says the following:

Theorem 1. Let $A$ be a local ring and let $P$ be a projective $A[T]$-module. If $P_{f}$ becomes a free $A[T]_{f}$-module, for some monic polynomial $f$ in $A[T]$, then $P$ is free. In other words, if $\mu\left(P_{f}\right)=\operatorname{rank}\left(P_{f}\right)(=\operatorname{rank}(P))$, then $\mu(P)=\operatorname{rank}(P)$.

By coupling Horrocks' Theorem with a recent result of Amit Roy [3, Theorem 1.1], one can even prove the following "qualitative" version of the Horrocks Theorem.

Theorem 1'. Let $A$ be a local ring and let $P$ be a projective $A[T]$-module. Then, for any monic polynomial $f$ in $A[T]$, the $A[T]$-module $P$ and the $A[T]_{f}$-module $P_{f}$ have the same minimal number of generators, i.e. $\mu(P)=\mu\left(P_{f}\right)$.

Let us first record the result of Amit Roy and then the proof of Theorem 1' will follow immediately.

THEOREM 2. Let $A$ be a local ring and let $P$ and $Q$ be projective $A[T]$-modules. Assume that $\operatorname{rank}(P)<\operatorname{rank}(Q)$. Suppose that $P_{f}$ is a direct summand of $Q_{f}$ for some monic polynomial $f$ in $A[T]$. Then $P$ is a direct summand of $Q$.

Proof of Theorem $1^{\prime}$. Let $f$ be a monic polynomial in $A[T]$. We know that $\mu\left(P_{f}\right) \leqslant \mu(P)$. So we need to prove that $\mu(P) \leqslant \mu\left(P_{f}\right)$. If $P_{f}$ is a free $A[T]_{f}$-module then $P$ is free by Horrocks' Theorem, and hence we get the desired conclusion. So we assume that $P_{f}$ is not free. Then $\operatorname{rank}\left(P_{f}\right)<\mu\left(P_{f}\right)$. Let $\mu\left(P_{f}\right)=n$. We can present $P_{f}$ as a direct summand of $\left(A[T]_{f}\right)^{n} \cong\left(A[T]^{n}\right)_{f}$. Since $\operatorname{rank}(P)=\operatorname{rank}\left(P_{f}\right)<$ $\operatorname{rank}\left(A[T]^{n}\right)$, Amit Roy's Theorem is applicable to give that $P$ is a direct summand of $A[T]^{n}$. Hence $\mu(P) \leqslant n$. Q.E.D.

We are naturally led to expect the following to be true.

Question. Let $A$ be a local ring and let $P$ and $Q$ be projective $A[T]$-modules. Suppose that $P_{f} \cong Q_{f}$ for some monic polynomial $f$ in $A[T]$. Is $P \cong Q$ ?

Received by the editors November 18, 1982.

1980 Mathematics Subject Classification. Primary 13C10. 
Let us recall a couple of definitions.

Definition. Let $R$ be a ring and let $P$ and $P^{\prime}$ be projective $R$-modules. Then $P$ and $P^{\prime}$ are called stably isomorphic if $P \oplus R^{n} \cong P^{\prime} \oplus R^{n}$ for some integer $n \geqslant 0$.

Definition. Let $R$ be a ring and let $P$ be a projective $R$-module. We say that $P$ satisfies the cancellation condition if any $P^{\prime}$ stably isomorphic to $P$ is in fact isomorphic to $P$.

Regarding the above question, in [3] it had been pointed out that $P$ and $Q$ are "fairly close" to being isomorphic. We shall list here some easy consequences.

(1) If $P$ or $Q$ is free then by Theorem $1, P \cong Q$.

(2) $P$ is a direct summand of $Q \oplus Q^{\prime}$ for any nonzero projective $A$ [T]-module $Q^{\prime}$.

Proof. A direct consequence of Theorem 2.

(3) $P$ and $Q$ are stably isomorphic.

Proof. Let $Q \oplus Q^{\prime}$ be a free $A[T]$-module for a suitable $Q^{\prime}$. Then $\left(P \oplus Q^{\prime}\right)_{f}$ is isomorphic to $\left(Q \oplus Q^{\prime}\right)_{f}$ and hence $P \oplus Q^{\prime}$ is free by (1). If $Q^{\prime} \oplus Q^{\prime \prime} \cong A[T]^{n}$ then it follows that $P \oplus A[T]^{n} \cong Q \oplus A[T]^{n}$.

(4) If $P$ or $Q$ contains a rank 1 direct summand then $P \cong Q$.

Proof. Suppose $P=P^{\prime} \oplus L$ where $L$ is of rank 1. Since $P_{f} \cong Q_{f}$, we get that $P_{f}^{\prime}$ is a direct summand of $Q_{f}$. Moreover, $\operatorname{rank}\left(P^{\prime}\right)<\operatorname{rank}(P)=\operatorname{rank}(Q)$. Therefore $P^{\prime}$ is a direct summand of $Q$, by Theorem 2. Say $Q \cong P^{\prime} \oplus L^{\prime}$. Now, as $P$ and $Q$ are stably isomorphic we obtain that $L$ and $L^{\prime}$ are stably isomorphic. But it is well known that rank 1 projective modules satisfy the cancellation condition. Therefore $L \cong L^{\prime}$ and hence $P \cong Q$.

(5) If $\operatorname{rank}(P)=1$ then $P \cong Q$.

Proof. Contained in (4).

(6) $P \oplus L \cong Q \oplus L$ for any rank 1 projective $A[T]$-module $L$.

Proof. Consequence of (4).

(7) $P$ and $Q$ have the same minimal number of generators.

Proof. Similar to that given for Theorem $1^{\prime}$.

The recent work of Plumstead [2] allows us to prove that the above question has a positive answer if $A$ is noetherian of dimension $\leqslant 1$. In fact, for this situation one does not need $A$ to be local. We first cite a theorem of Plumstead.

THEOREM 3. Let $R$ be a noetherian polynomial ring of dimension $d$. Then every projective $R$-module of rank $\geqslant d$ satisfies the cancellation condition.

Now let $A$ be a noetherian ring and let $R=A[T]$. Let $P$ and $Q$ be projective $R$-modules such that $P_{f} \cong Q_{f}$ for some monic polynomial $f$ in $R$. We can appeal to the Quillen-Suslin affine Horrocks' Theorem and conclude that $P$ and $Q$ are stably isomorphic. If $\operatorname{dim}(A) \leqslant 1$ or equivalently $\operatorname{dim}(R) \leqslant 2$, then $P$ and $Q$ would be isomorphic, by Theorem 3 and the fact that rank 1 projectives satisfy the cancellation condition.

This remark came into existence out of a discussion with Paul Eakin. In fact Paul Eakin had, several years ago, posed Theorem $1^{\prime}$ as a question.

It would be interesting to prove Theorem $1^{\prime}$ without the assumption that $A$ is a local ring. 


\section{REFERENCES}

1. G. Horrocks, Projective modules over an extension of a local ring, Proc. London Math. Soc. 14 (1964), 714-718.

2. B. R. Plumstead, The conjectures of Eisenbud and Evans, dissertation, University of Chicago, August. 1979.

3. Amit Roy, Remarks on a result of Roitman, Tata Institute, preprint.

Department of Mathematics, Pennsylvania State University, Mont alto Campus, Mont Alto, Pennsylvania 17237 